\title{
Effect of the Electrode Material on Ignition of Ethylene-Air Mixtures
}

\author{
Abdeldjalil Reguig, Jason S. Damazo, Eddie Kwon, and Deanna A. Lacoste
}

\begin{abstract}
This paper presents an experimental quantification of the ignition risk of flammable mixtures in conditions relevant to industry and aviation environment. More specifically, the effect of resistive material on ignition of flammable mixtures was investigated by applying a 500-ns duration high-voltage pulse between pin-to-pin electrodes located across a jet flow of ethylene and air, at atmospheric pressure. Fuel-rich, stoichiometric and fuel-lean equivalence ratios were considered. A parametric study of the effect of the applied voltage and electrode material was performed. The electrical energy deposited in the discharges was determined from current and voltage measurements. Direct visualization of the sparks and the ignition kernel was performed in order to better understand the early stages of the ignition process. The results showed that the use of highly-resistive electrodes compared to metallic electrodes (i) could significantly reduce the energy deposition in the interlectrode area, and (ii) could increase the minimum ignition energy. Therefore, the use of highly-resistive material wires instead of metallic wires could offer safer conditions with respect to the ignition hazard in flammable environments.
\end{abstract}

Index Terms-Minimum ignition energy Nanosecond discharge Flammable mixtures Highly-resistive material

\section{INTRODUCTION}

With the evolution of methods, designs and materials, the risks of accidental ignition in industrial environments are changing. Quantifying these new risks is of major importance in industry and aviation. In addition, new materials and system architectures may be explored to help reduce the threat of ignition source generation. In any flammable gas mixture, an unexpected spark can be the reason for a dramatic accident. The energy, power and volume of a spark are the most important parameters characterizing the ignition threat [1, 2, 3]. All these parameters result from the electrical properties of the configuration leading to the undesired spark. Therefore, any changes in the electrical properties of the materials as well as in their geometry can affect the risk of accidental ignition.

Numerous research groups have focused their efforts investigating the mitigation of inadvertent ignition in aircraft environments [4, 5, 6, 7, 8, 9]. Along with several standard

This paper was submitted for review on April 15, 2020. This work was supported by The Boeing Company and the King Abdullah University of Science and Technology.

Abdeldjalil Reguig is with the King Abdullah University of Science and Technology, Thuwal 23955-6900, Saudi Arabia (abdeldjalil.reguig@kaust.edu.sa).

Jason Damazo is with The Boeing Company, Boeing Research and Technology, Seattle, WA, USA (jason.s.damazo@boeing.com).

Eddie Kwon is with Narion Corporation, Seattle, WA, USA (eddie.kwon@narioncorp.com).

Deanna Lacoste is with the King Abdullah University of Science and Technology, Clean Combustion Research Center, Thuwal 23955-6900, Saudi Arabia (deanna.lacoste@kaust.edu.sa). test methods [10, [11, 12], the results of these studies have been used to efficiently control ignition by undesired sparks in aircraft. However, all these studies were considering simple electrode models, being either a conductor (metal) or a dielectric.

Recently, several studies have shown that the use of resistive electrodes could significantly affect the electrical breakdown, compared to metallic electrodes [13, 14, 15, 16, 17, 18]. For example, Laroussi et al. [13] introduced the terms of resistive barrier discharges (RBD), and showed that resistive electrodes could avoid the transition from a spark to an arc discharge. More recently, Reguig et al. [18] showed that, in ambient air, compared to copper electrodes, electrodes made of highly resistive material could significantly affect both the breakdown mechanism and the energy deposition in the discharge. Thus, resistive materials could be used for equipment parts or wire used for data and low power transmission to reduce the likelihood of undesired ignitions. However, to the best of our knowledge, the effect of resistive material on ignition of flammable mixture has never been investigated. This is the purpose of the present study.

Evaluation of the ignition threat was performed with ethylene-air mixtures. Ethylene-air is commonly used in the aerospace industry as a repeatable and conscervative surrogate for jet fuel [6, 19]. A simple pin-to-pin electrode configuration, with a gap distance of a $2 \mathrm{~mm}$, has been selected. The $2 \mathrm{~mm}$ gap distance represents the minimum distance for ignition threats in the aerospace industry. This canonical configuration is representative of realistic configurations for accidental sparks, for example when a grounding cable has been inadvertently cut or when one system is elevated to a higher voltage compared to a nearby grounded conductor in aircraft lightning environments. It will also ease further comparisons with numerical simulations. Finally, the electrical discharges were produced by applying a single high-voltage pulse of 500-ns duration. This discharge duration is representative of electrostatic discharges, which usually last between $1 \mathrm{~ns}$ and $1 \mathrm{~ms}$ [20, 9], and it was chosen to represent a sufficiently long time duration for aircraft in direct and indirect lightning environments.

\section{EXPERIMENTAL SETUP AND PROCEDURE}

The experimental setup used in this study was comprised of: a jet burner, a nanosecond spark ignition system, and electrical and optical diagnostics. Figure 1 presents a schematic of the experimental setup and a photograph of the two electrodes installed on top of the burner. 


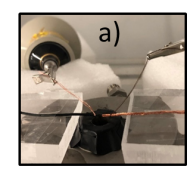

b)
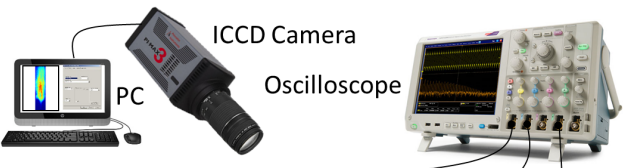

HV Pulser

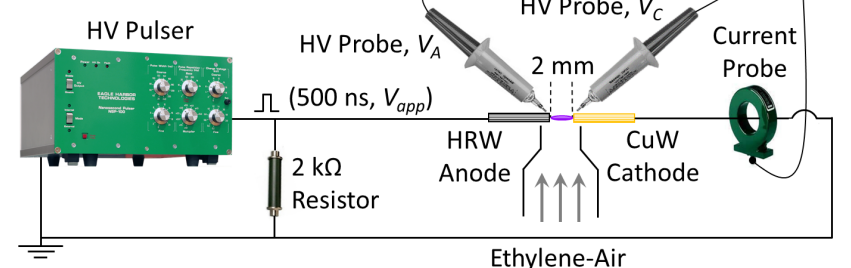

Fig. 1. a) Photograph of the electrodes: on the left, the HRW-50mm anode and and on the right, the $\mathrm{CuW}$ cathode, and b) schematic of the experimental setup.

The ignition tests were conducted in a vertical cylindrical jet burner of 10-mm diameter, at atmospheric pressure and $293 \mathrm{~K}$ of temperature. The flow rates of air and ethylene were regulated using mass flow controllers (Brooks SLA 58 Series), not shown in Fig. 1 Before entering the test section, a perfect mixture of the air and ethylene was ensured via a mixing box located $1 \mathrm{~m}$ upstream of the burner outlet. Three equivalence ratios, $\phi$, were investigated: a fuel-rich condition, $\phi=1.2$, a stoichiometric condition, $\phi=1$, and a fuel-lean condition, $\phi=0.88$. For all cases, the bulk velocity, $V_{b u l k}$, at the outlet of the burner was $21 \mathrm{~m} / \mathrm{s}$, corresponding to a Reynolds number of about 14,000 . Note that the effects of flow velocity or turbulence intensity were not investigated in this study.

The electrodes had a pin-to-pin configuration, with a gap distance of $2 \mathrm{~mm}$, centered on the axis of the burner, $5 \mathrm{~mm}$ above the burner outlet. Several arrangements of electrode pairs were used. For each pair, one electrode was a copper wire (referred to as $\mathrm{CuW}$ ) of $50 \mathrm{~mm}$ length and $0.7 \mathrm{~mm}$ diameter, while the second electrode was another $\mathrm{CuW}$, another $\mathrm{CuW}$ in series with a $50-\mathrm{k} \Omega$ resistor, or a highly-resistive wire of $0.7-$ mm diameter (referred to as HRW) of various lengths. Table I summarizes the tested configurations. The material used for the HRW was not a commercial material and no reference can be provided. It was made of nylon with a uniformly distributed proprietary formula to increase conductivity. Its electrical characterization as well as its effect on the electrical characteristics of discharges in air at atmospheric condition can be found in [18]. The average resistivity of the HRW was $7.3 \times 10^{-2} \Omega . \mathrm{m}$, and its capacitance with respect to the grounded test apparatus was $1 \mathrm{pF}$.

The anode was connected to a high-voltage power supply (Eagle Harbor Technologies, model NSP-120-20-P). A single high-voltage pulse of 500-ns duration was applied for each ignition test. The applied voltage was varied from $9 \mathrm{kV}$, lower voltage at which a discharge was produced, to $20 \mathrm{kV}$, maximal voltage of the power supply. The cathode was groun-ded. For impedance matching, a non-inductive $2 \mathrm{k} \Omega$ resistor (Kanthal) was inserted in parallel with the electrodes. This resistor minimized the signal reflection from the load and produced a reasonably square-shaped high-voltage pulse.

In order to characterize the electrical discharges, simulta-
TABLE I

SUMMARY OF THE ELECTRODE CONFIGURATIONS. CUW STANDS FOR COPPER WIRE AND HRW STANDS FOR HIGHLY-RESISTIVE WIRE. FOR ALL PAIRS OF ELECTRODES, THE GAP DISTANCE WAS $2 \mathrm{MM}$.

\begin{tabular}{lccccc}
\hline Material & $\begin{array}{c}\text { Anode } \\
\text { Length } \\
(\mathrm{mm})\end{array}$ & $\begin{array}{c}\text { Resistance } \\
(\mathrm{k} \Omega)\end{array}$ & Material & $\begin{array}{c}\text { Cathode } \\
\text { Length } \\
(\mathrm{mm})\end{array}$ & $\begin{array}{c}\text { Resistance } \\
(\mathrm{k} \Omega)\end{array}$ \\
\hline $\mathrm{CuW}$ & 50 & 0 & $\mathrm{CuW}$ & 50 & 0 \\
& & & $\mathrm{CuW}$ & 50 & 50 \\
& & & HRW & 50 & 12 \\
$\mathrm{CuW}$ & 50 & 0 & HRW & 150 & 25 \\
& & & HRW & 300 & 50 \\
& & & HRW & 500 & 120 \\
$\mathrm{CuW}$ & 50 & 50 & & & \\
HRW & 50 & 12 & & & \\
HRW & 150 & 25 & CuW & 50 & 0 \\
HRW & 300 & 50 & & & \\
HRW & 500 & 120 & & & \\
\hline
\end{tabular}

neous current and voltage measurements were performed (see Fig. 1p). Two similar high-voltage probes (Tektronix, model P6015A) were connected to the electrodes within $1 \mathrm{~mm}$ from the electrode tip (see Fig. 11). The gap voltage, $V_{g}$, was obtained by:

$$
V_{g}=V_{A}-V_{C}
$$

where $V_{A}$ is the voltage measured between the tip of the anode and the ground and $V_{C}$ is the voltage measured between the tip of the cathode and the ground. Note that when the cathode was the $\mathrm{CuW}$ without resistor, $V_{C}=0 \mathrm{~V}$.

In addition, a current transformer (Pearson, model 6585) measured the total discharge current, $I_{t o t}$. Both current and voltage waveforms were recorded using an oscilloscope (Tektronix, model DPO5204B, $2 \mathrm{GHz}$ bandwidth). The energy deposition in the gap, $E_{g}$, was determined by integrating the product of the gap voltage and the total current over the discharge duration, $\tau$ :

$$
E_{g}=\int_{0}^{\tau} V_{g}(t) I_{t o t}(t) d t
$$

In parallel to the electrical characterization of the discharges, the ignition events were investigated by direct visualization. The ignition events were assessed by taking $3 \mathrm{~s}$ timeintegrated images with an intensified CCD camera (Prin-ceton Instruments, PIMAX 3), equipped with an UV lens (105F/4). Figure 2 presents examples of images taken for successful ignitions (Figs. $2 \mathrm{a}$ and $2 \mathrm{p}$ ) and ignition failures (Figs. 2k and $2 \mathrm{~d})$. The temporal evolution of the ignition kernels was also investigated. As the maximal repetition rate of the camera was only $20 \mathrm{~Hz}$, single-shot phase-locked imaging was performed with a constant exposure time of $10 \mu \mathrm{s}$. The camera and the discharges were synchronized with a pulse delay generator (Berkley Nucleonix, model 575), not shown in Fig. 1

As expected for conditions close to the ignition threshold, Fig. 2 also highlights that, for a given experimental condition, the spark could sometimes be an ignition source and sometimes not (see for example Figs. 2 a and 22). Consequently, for each experimental condition, the ignition tests were repeated at least 20 times in order to determine an ignition probability. 

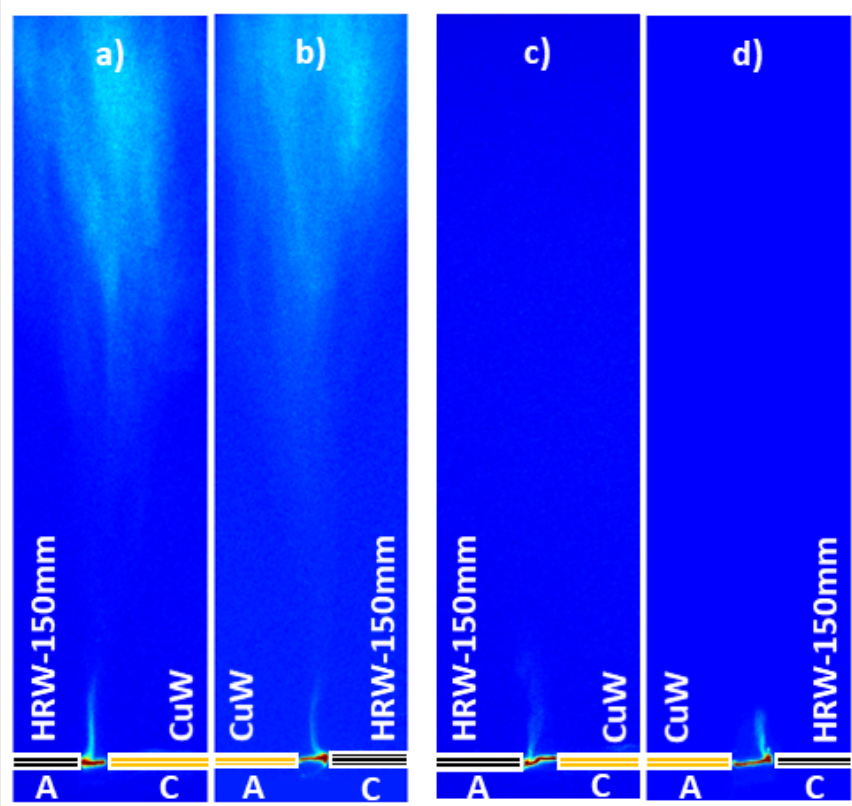

Fig. 2. Examples of visualization of spark discharges with ignition (a and b) and without ignition (c and d), for an electrode pair CuW-50mm, HRW$150 \mathrm{~mm}$, and for both polarity of $10 \mathrm{kV}$ applied voltage (A stands for anode and $\mathrm{C}$ for cathode). Exposure time of $3 \mathrm{~s}$.

A minimal delay of $20 \mathrm{~s}$ between two consecutive tests was considered. The minimum ignition energy (MIE) [2, 21], was here defined as the minimal energy needed for an ignition probability of at least $5 \%$ (one successful ignition from twenty sparks).

\section{RESULTS AND DISCUSSION}

In this section, first the effect of the gas mixture composition on the electrical characteristics of the discharges and the probability of ignition is presented. Then, the MIE obtained for all electrode configurations are detailed and discussed. Finally, the temporal evolution of the ignition kernels are analyzed.

\section{A. Effects of the Gas Mixture Composition}

Changes in the gas mixture composition could significantly affect both the breakdown and the electrical characteristics of the subsequent discharge. Figure 3 compares the gap voltage, $V_{g}$, the total current, $I_{t o t}$ and the energy deposited in the gap, $E_{g}$, for a discharge produced by a $500-\mathrm{ns}$ pulse of $16-\mathrm{kV}$ applied voltage, (a) in air, and (b) in a stoichiometric mixture of ethylene and air. The electrodes were a pair of HRW-150mm (anode) and $\mathrm{CuW}$ (cathode).

In both cases, the voltage and current waveforms were relatively similar. The breakdown occured at about $9.5 \mathrm{kV}$ and the peak of current was about $1 \mathrm{~A}$. However, after the first peak of current, in air (Fig. 3 3 ), the current remained almost constant with a long voltage plateau of $V_{g}=1 \mathrm{kV}$ for about $300 \mathrm{~ns}$. The corresponding energy was about $0.8 \mathrm{~mJ}$. In the stoichiometric mixture of ethylene and air (Fig. 3 3 ), the current dropped significantly after the first peak resulting in a relatively high $V_{g}$ of about $4 \mathrm{kV}$. The plateau of $V_{g}=1 \mathrm{kV}$
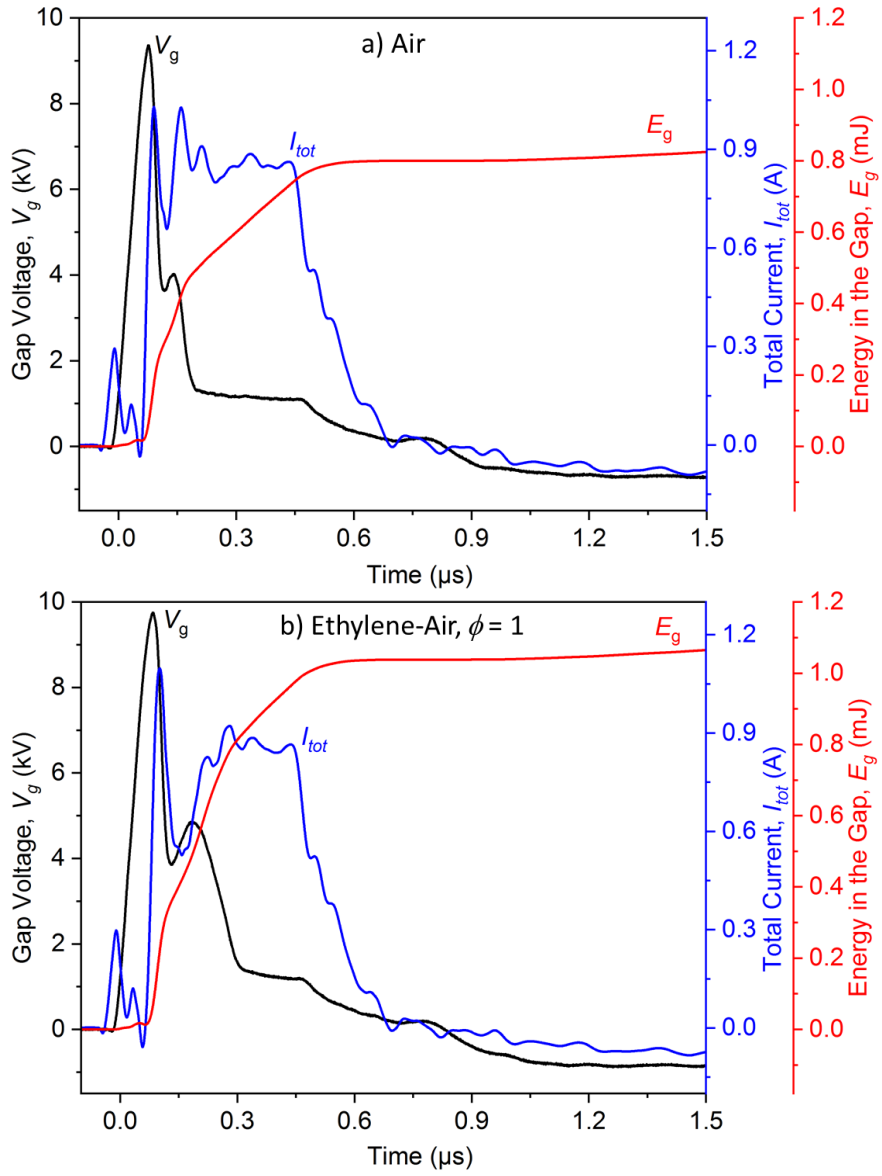

Fig. 3. Examples of temporal evolution of gap voltage, total current and corresponding deposited energy, for a HRW-150mm anode and a CuW cathode, and an applied voltage of $V_{a p p}=16 \mathrm{kV}$, a) in an air flow, and b) in a stoichiometric ethylene-air flow.

lasted about $150 \mathrm{~ns}$, and the corresponding energy was slightly more than $1 \mathrm{~mJ}$, i.e., about $20 \%$ higher than in air. This higher energy deposition in ethylene-air mixtures compared to air was systematically observed for all conditions. This difference is consistent with the fact that hydrocarbon gases have a higher permittivity than air [22]. However, for the three equivalence ratio investigated $(0.88,1$ and 1.2$)$, no significant difference in the electrical properties of the discharges could be observed. Therefore, comparisons of the ignition probabilities for the three gas mixtures as a function of the applied voltage are meaningful.

Figure 4 presents the ignition probability as a function of the applied voltage, for the three mixtures of ethylene and air considered ( $\phi=0.88$, stoichiometric and $\phi=1.2)$, for a HRW$150 \mathrm{~mm}$ anode and a $\mathrm{CuW}$ cathode. For an applied voltage of $9 \mathrm{kV}$, no ignition event could be obtained, while for an applied voltage higher than $17 \mathrm{kV}, 100 \%$ of ignition was obtained for the three mixtures. In between, as expected, the rich mixture, $\phi=1.2$, had the highest ignition probability, while the lean mixture, $\phi=0.88$, had the lowest. For safety problems, even an ignition probability as low as $5 \%$ has to be considered, as it could be the source of a catastrophic accident. In the next section, the effect of the electrode configurations on the MIE, defined as the energy at which a probability of ignition of at 


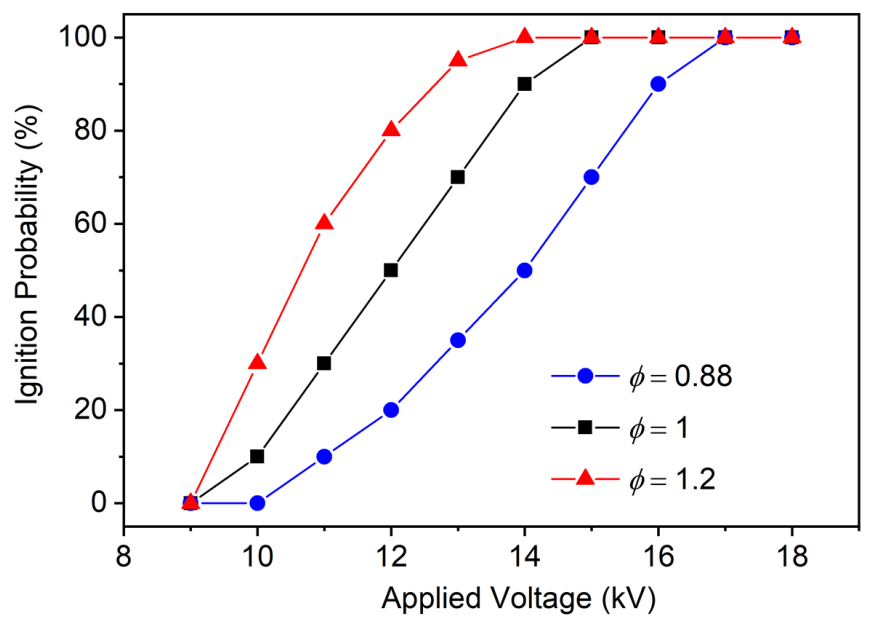

Fig. 4. Ignition probabilities as a function of the applied voltage for a HRW$150 \mathrm{~mm}$ anode and a $\mathrm{CuW}$ cathode, for different equivalence ratios of ethyleneair mixtures.

least $5 \%$ was observed, is discussed.

\section{B. Effect of the Electrodes on the MIE}

Figure 5 presents for all electrode configurations, the energy deposited in the gap, $E_{g}$, as a function of the applied voltage, in flows of stoichiometric $(\phi=1)$, ethylene and air mixture at atmospheric conditions, for a gap distance of $2 \mathrm{~mm}$. The error bars correspond to the standard deviation calculated from 10 different measurements. The conditions for which ignition could be obtained are reported with solid symbols, while the conditions for which no ignition was observed are reported with empty symbols.

For all conditions, the energy deposited in the gas increased linearly with the applied voltage. In addition, for a similar pair of electrodes, the energy deposited in the gap is minimally affected by the polarity. This is in agreement with our previous work for discharges in air [18].

For $\mathrm{CuW}$ without resistor (black squares), HRW-50mm (blue circles), and HRW-150mm (brown diamonds), anodes (Fig. 5a), or cathodes (Fig. 5b), as soon as a spark could be obtained (for an applied voltage higher than $9 \mathrm{kV}$ ), ignition could also be observed. Therefore, for these three conditions, the MIE was widely overestimated by the method used. Nevertheless, it is interesting to note that for HRW-150mm cathode, the MIE was about $450 \mu \mathrm{J}$. This value is of the same order than the MIE from the literature for quiescent stoichiometric ethylene-air mixtures at ambient conditions, for a gap distance of $1.3 \mathrm{~mm}: 105 \mu \mathrm{J}$ [23]. Of course, the MIE is also a function of the spark duration, which is not discussed here.

For a HRW-500mm (violet triangles), anode or cathode, even if sparks could be obtained for applied voltages higher than $11 \mathrm{kV}$, no ignition was observed and the MIE could not be determined. These results highlight the effect of the electrode material and length on the ignition hazard. By increasing the wire length from 50 to $500 \mathrm{~mm}$, the equivalent resistance of the electrodes were increased from 12 to $120 \mathrm{k} \Omega$ (see TableI). Therefore, for the HRW-500mm electrode, a large fraction of
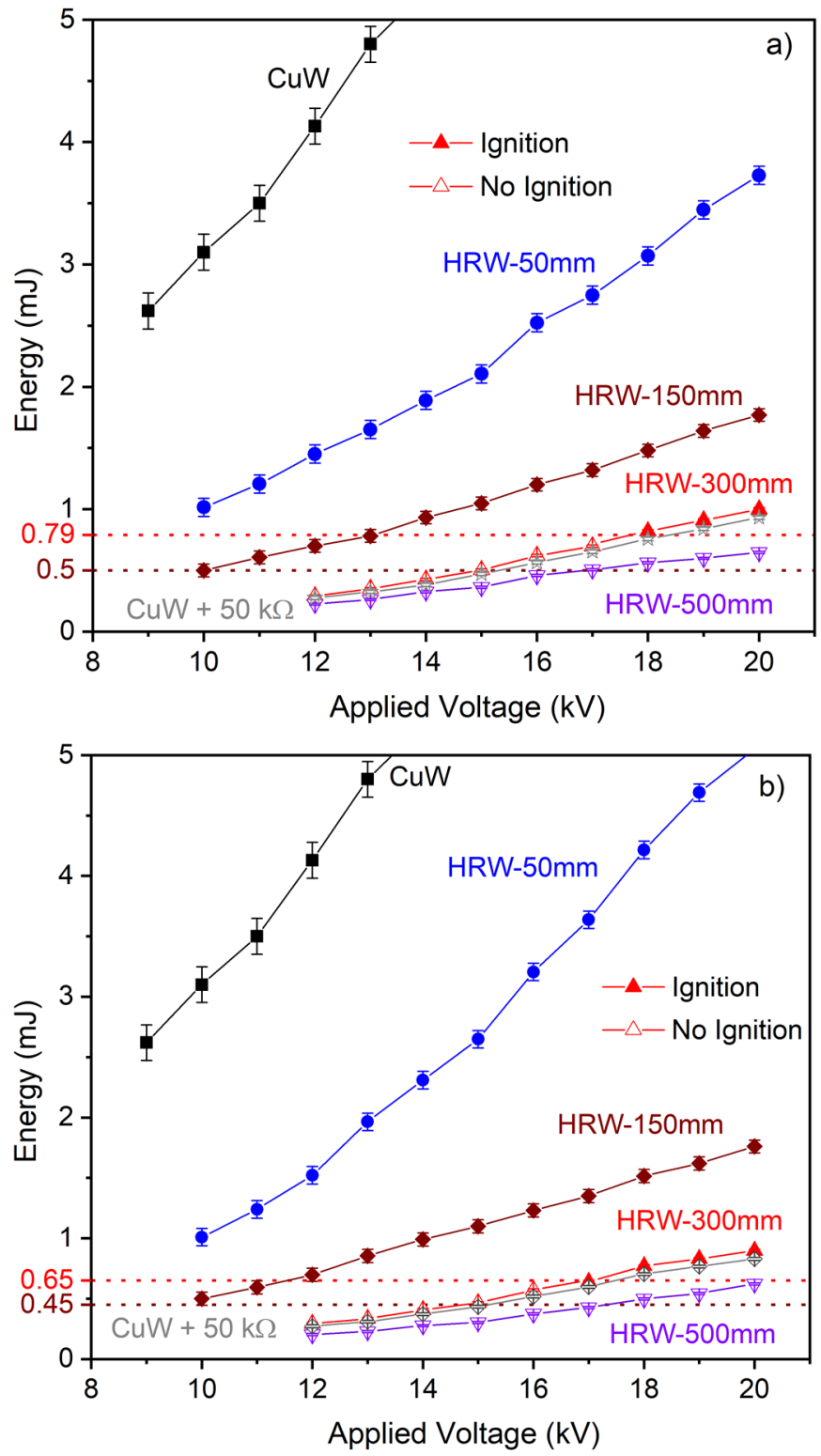

Fig. 5. Evolution of the the energy deposited into the gas, $E_{g}$, as function of the applied voltage with the respective MIE for: a) different wire anodes, and b) different wire cathodes.

the available electrical energy was lost by Joule heating of the resistive wire, and not deposited in the discharge. As a consequence, the mixture could not be ignited.

An interesting case is for the HRW-300mm electrode (red triangles). With this electrode, for an applied voltage between 12 and $16 \mathrm{kV}$, sparks could be obtained but no ignition was observed. In the case of the HRW-300mm anode (Fig. 5a ), the MIE was $790 \mu \mathrm{J}$, corresponding to an applied voltage of $18 \mathrm{kV}$, and for the case of the HRW-300mm cathode (Fig. 5 5 ), the MIE was $650 \mu \mathrm{J}$ for an applied voltage of $17 \mathrm{kV}$. In both cases, the MIE was significantly higher than for HRW-150mm (500 and $450 \mu \mathrm{J}$, respectively). As the pulse duration as well as all other parameters where kept constant for both HRW lengths, it would have been expected that the MIE would be the same. This difference on the ignition hazard could be explained by a 

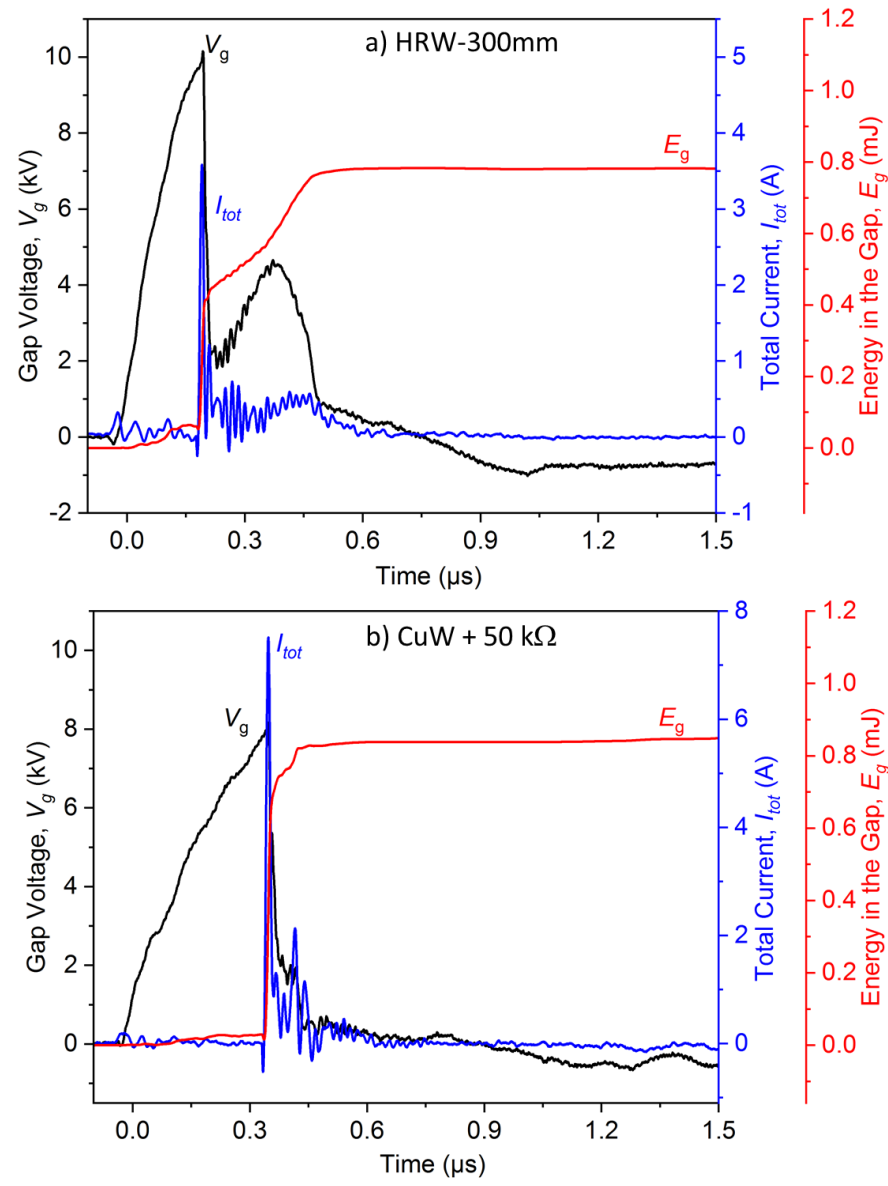

Fig. 6. Temporal evolution of gap voltage, $V_{g}$, total current, $I_{t o t}$, and the corresponding deposited energy in the gap, $E_{g}$, for $V_{a p p}=19 \mathrm{kV}$ and a) for a HRW-300mm anode, and b) for an anode made of $\mathrm{CuW}$ with a $50 \mathrm{k} \Omega$ resistor.

difference in the discharge power density, i.e., in the discharge volume and/or power.

In order to further investigate the electrode effect on the ignition hazard, tests were performed with a $\mathrm{CuW}$ in series with a $50 \mathrm{k} \Omega$ resistor (grey stars), corresponding to the equivalent resistance of the HRW-300mm. In this case, even though the energies deposited in the gap were comparable with those obtained with HRW-300mm, no ignition was observed; this held true in both polarities (see Fig. 5).

For an applied voltage of $19 \mathrm{kV}$, the temporal evolution of the gap voltage, total current and energy deposited in the gap for these two electrodes can be compared in Fig. 6 . Even though $E_{g}$ was similar for both electrodes, the maximal intensities and the shapes of $V_{g}$ and $I_{t o t}$ were very different. For the HRW-300mm anode, the maximal $V_{g}$ was $10 \mathrm{kV}$ and a second peak of $4 \mathrm{kV}$, about $300 \mathrm{~ns}$ after the breakdown, can be noticed. For the $\mathrm{CuW}$ with a $50-\mathrm{k} \Omega$ resistor, the breakdown occurred at a lower voltage of $8 \mathrm{kV}$ and no second voltage peak is observed. On the other hand, the peak of $I_{\text {tot }}$ was higher for the $\mathrm{CuW}$ with resistor $(7.5 \mathrm{~A})$, than for the HRW$300 \mathrm{~mm}(3.5 \mathrm{~A})$. These differences could be explained by the capacitive effect of the HRW. After a first current peak, during the remaining time of the applied pulse, the wire stored a
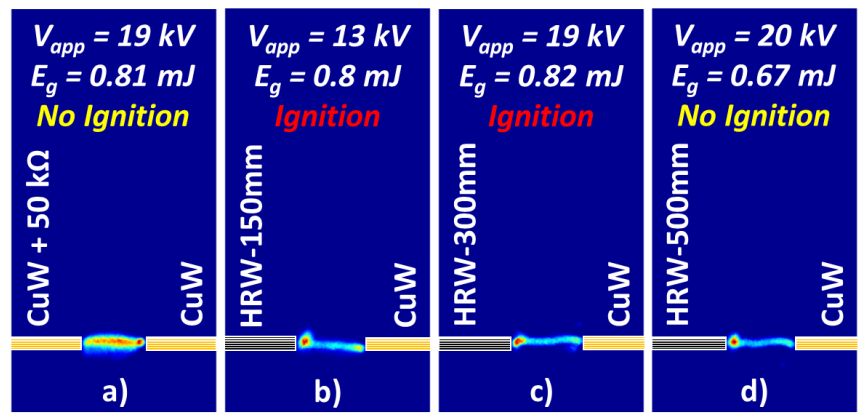

Fig. 7. Direct visualization of the discharges obtained in stoichiometric ethylene-air flow, with a gap distance of $2 \mathrm{~mm}$ for a) an anode made of $\mathrm{CuW}$ with a $50-\mathrm{k} \Omega$ resistor, b) a HRW-150mm anode, c) a HRW-300mm anode, and d) a HRW-500mm anode. Exposure time of $10 \mu \mathrm{s}$.

considerable amount of charge and the voltage increased again. For the $\mathrm{CuW}$ with resistor, all the electric energy was released as soon as the discharge was generated in the gap. In this case, the current waveform was characterised by a single-pulse and the gap voltage fell down rapidly. Consequently, for the HRW$300 \mathrm{~mm}, E_{g}$ was released in about $400 \mathrm{~ns}$, corresponding to an average power of $2 \mathrm{~kW}$, while for $\mathrm{CuW}$ with resistor, $E_{g}$ was released in less than $100 \mathrm{~ns}$, which corresponds to an average power of $8 \mathrm{~kW}$. In the absence of other effects, this higher power should be more efficient for ignition, which does not support the results presented in Fig. 5. Therefore, different discharge volumes might be responsible for the different MIE obtained with the different electrodes.

Figure 7 presents single-shot direct visualizations of the discharges, recorded with an exposure time of $10 \mu \mathrm{s}$, i.e., collecting all the light emission from the 500-ns discharges. The discharges were obtained for a) an anode made of $\mathrm{CuW}$ with a $50-\mathrm{k} \Omega$ resistor, b) a HRW-150mm anode, c) a HRW$300 \mathrm{~mm}$ anode, and d) a HRW-500mm anode, for similar $E_{g}$. Note that for HRW-500mm, only $E_{g}=0.67 \mathrm{~mJ}$ could be achieved with the maximal applied voltage of $20 \mathrm{kV}$. Ignition could be obtained for HRW-150mm and HRW-300mm (Figs. 7b and 7k, respectively), and no ignition was observed for $\mathrm{CuW}$ with resistor and HRW-500mm (Figs. 7a and 7d, respectively). These pictures shows that the discharge volume and intensity were different for $\mathrm{CuW}$ with resistor and for the HRW electrodes. For the $\mathrm{CuW}$ with resistor, the discharge was homogeneous in the interelectrode gap, while for the HRW electrodes, the discharges featured an intense spot close to the anode and a relatively mild discharge channel across the gap. This intense localized light emission close to the electrode is an indicator that the main part of the electrical energy is deposited in this area, where the energy density is therefore much higher than in the case of $\mathrm{CuW}$. In order to verify if this intense spot is associated with the ignition process, phase-locked images of ignition events are analyzed in the next section.

\section{Temporal Evolution of the Ignition Kernels}

Figures 8 and 9 present the temporal evolution of the light emission during ignition events obtained in a stoichiometric 


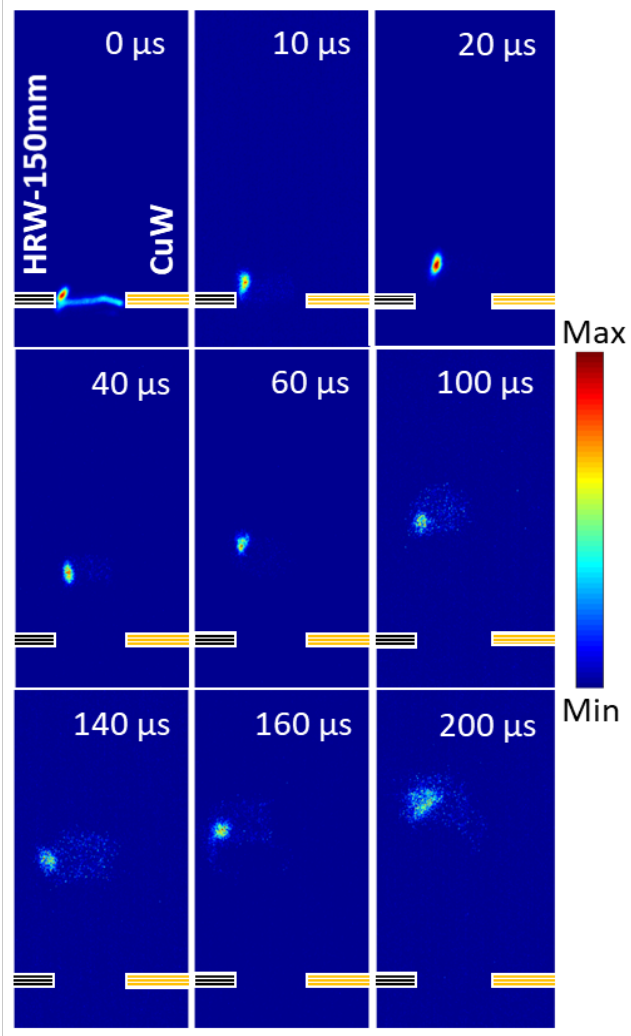

Fig. 8. Phase-locked images of the ignition of a stoichiometric flow of ethylene and air, obtained for an applied voltage of $16 \mathrm{kV}$, with a HRW$150 \mathrm{~mm}$ anode. Exposure time of $10 \mu \mathrm{s}$.

ethylene-air flow for an applied voltage of $16 \mathrm{kV}$, with a HRW$150 \mathrm{~mm}$ anode and a HRW-150mm cathode, respectively. The colorbars are adapted to the intensities of each image in order to clearly follow the ignition process. Time zero corresponds to the beginning of the 500-ns voltage pulse.

First, it can be noticed that the development of the ignition kernel was similar for both configurations. For both polarities, it started from the intense discharge zone, close to the HRW electrode, and it was convected by the gas flow, while progressively expanding. At $200 \mu \mathrm{s}$, the ignition kernels had a diameter of about $1 \mathrm{~mm}$, and were convected by about $6 \mathrm{~mm}$ above the electrodes. The ignition was obviously starting from the intense spot of the discharge, and not from the filament crossing the gap. This result highlights that the effect of the electrode material on the discharge morphology can have a significant impact on the ignition hazard. For similar geometries and deposited energies, if the breakdown and discharge processes are affected by the electrode material, the resulting difference in local power density could significantly affect the ignition probability.

\section{CONCLUSION}

In this study, the effect of electrode properties on the ignition probability and minimum ignition energy (MIE) of ethylene-air flows have been experimentally investigated. Electrodes made of copper and of highly-resistive material of different lengths, therefore of different equivalent resistance, were compared. Main findings were:

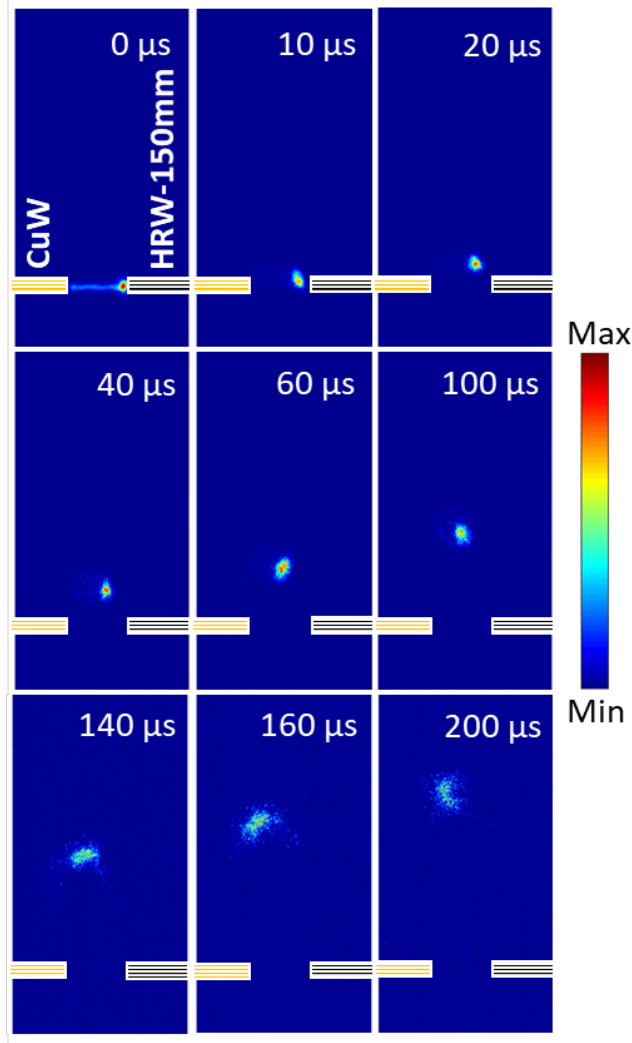

Fig. 9. Phase-locked images of the ignition of a stoichiometric flow of ethylene and air, obtained for an applied voltage of $16 \mathrm{kV}$, with a HRW$150 \mathrm{~mm}$ cathode. Exposure time of $10 \mu \mathrm{s}$.

- Compared to air, the presence of ethylene in the gas flow increased the energy deposited in the discharge by about $20 \%$, for any electrode configuration. This could be attributed to a higher permittivity of ethylene compared to air.

- Electrodes made of highly-resistive material efficiently decreased the deposited energy compared to copper electrodes. In addition, increasing the length of the highlyresistive electrode also increased the MIE, leading to a safer configuration.

- However, the highly-resistive material could also affect the discharge morphology in the gap, leading to an increase in the ignition hazard, compared to a copper electrode in series with a similar equivalent resistor. Therefore, for evaluating the ignition threat from various material and electrical property systems, an equivalent system evaluation is insufficient. Finer details in the configuration needs to be investigated to get an accurate determination of the threat.

These results are promising for the control of ignition hazard by the electrical properties of cables. However, they also emphasize that for a given electrode configuration, the determination of the deposited power and energy is not enough to predict ignition. However, a coupled electrical and optical characterization of a discharge should be sufficient to correlate ignition hazard and electrode properties. These results can be used by safety engineers for evaluation of the ignition threat, 
for example in the case of a broken cable.

\section{REFERENCES}

[1] M. Blanc, P. Guest, G. von Elbe, and B. Lewis, "Ignition of explosive gas mixtures by electric sparks: Iii. minimum ignition energies and quenching distances of mixtures of hydrocarbons and ether with oxygen and inert gases," Symposium on Combustion and Flame, and Explosion Phenomena, vol. 3, pp. 363-367, 1948.

[2] B. Lewis and G. von Elbe, Combustion, Flames and Explosions of Gases, 2nd ed. Academic Press, New York, USA, 1961.

[3] V. Babrauskas, Ignition handbook: Principles and applications to fire safety engineering, fire investigation, risk management and forensic science. Fire Science Publishers, Issaquah, WA, USA, 2003.

[4] W. Hall, "Electrostatic dischargers for aircraft," Journal of Applied Physics, vol. 18, 8, pp. 759-765, 1947.

[5] R. V. Dolah, M. Zabetakis, D. Burgess, and G. Scott, "Ignition or the flame-initiating process," Fire Technology, vol. 1, pp. 32-42, 1965.

[6] P. Dagaut and M. Cathonnet, "The ignition, oxidation, and combustion of kerosene: A review of experimental and kinetic modeling," Progress Energy Combust. Sci., vol. 32, pp. 48--92, 2006.

[7] S. Bane, "Spark ignition: Experimental and numerical investigation with application to aviation safety," Ph.D. dissertation, California Institute of Technology, USA, 2010.

[8] S. Bane, J. Ziegler, P. Boettcher, S. Coronel, and J. Shepherd, "Experimental investigation of spark ignition energy in kerosene, hexane, and hydrogen," J. Loss Prevent. Proc., vol. 26, pp. 290-294, 2013.

[9] J. Gieras, "Electrical ignition of fuel-air mixture in aircraft fuel tanks," Przeglad Elektrotechniczny, vol. 89, pp. 17--24, 2013.

[10] F. R. DOT/FAA/CT-94/74, “Aircraft fuel system lightning protection design and qualification test procedures development," U.S. Dept. of Transportation Federal Aviation Administration, USA, Tech. Rep., 1994.

[11] ARP5416, "Aerospace recommended practice aircraft lightning test methods," SAE International, USA, Tech. Rep., 2005.

[12] E582-07, "Standard test method for minimum ignition energy and quenching distance in gaseous mixtures," American Society for Testing and Materials International, USA, Tech. Rep., 2009.

[13] M. Laroussi, I. Alexeff, J. Richardson, and F. F. Dyer, "The resistive barrier discharge," IEEE Trans. Plasma Sci., vol. 30, pp. 158-159, 2002.

[14] K. Stephan, S. Ghimire, R. Smith, L. Komala-Noor, and N. Massey, "Transverse stabilization of atmosphericpressure dc glow plasma in air with resistive barrier," IEEE Trans. Plasma Sci., vol. 39, pp. 1919-1926, 2011.

[15] M. Thiyagarajan, A. Sarani, and X. Gonzales, "Atmospheric pressure resistive barrier air plasma jet induced bacterial inactivation in aqueous environment," J. Appl. Phys., vol. 113, p. 093302, 2013.
[16] X. Wang, Z. Li, J. Zeng, X. Zhang, and L. Lei, "Improvement of atmospheric water surface discharge with water resistive barrier," Plasma Chem. Plasma Process., vol. 33, pp. 691-705, 2013.

[17] K. Stephan and G. Sheleg, "Directional propagation of resistive-barrier discharge above water," IEEE Trans. Plasma Sci., vol. 43, pp. 501-505, 2015.

[18] A. Reguig, B. Ramljak, K. Chatelain, J. Damazo, E. Kwon, and D. Lacoste, "Comparison of electrical breakdowns produced by a nanosecond high-voltage pulse applied to metallic and composite material electrodes," IEEE Trans. Plasma Sci., vol. 47, pp. 46834690, 2019.

[19] X. Wu, Z. Yang, X. Wang, and Y. Lin, "Experimental and theoretical study on the influence of temperature and humidity on the flammability limits of ethylene (r1150)," Energy, vol. 52, pp. 185-191, 2013.

[20] H. Fu, Y. Xie, and J. Zhang, "Analysis of corona discharge interference on antennas on composite airplanes," IEEE Trans. Electromagn. Compat., vol. 50, pp. 822827, 2008

[21] E. Litchfield, Minimum ignition-energy concept and its application to safety engineering. Technical report, Bureau of Mines Report of Investigations 5671, 1960.

[22] J. Schmidt and M. Moldover, "Dielectric permittivity of eight gases measured with cross capacitors," Int. J. Thermophysics, vol. 24, pp. 375-403, 2003.

[23] A. Wahner, G. Gramse, T. Langer, and M. Beyer, "Determination of the minimum ignition energy on the basis of a statistical approach," J. Loss Prevent. Proc., vol. 26, pp. 1655-1660, 2013.

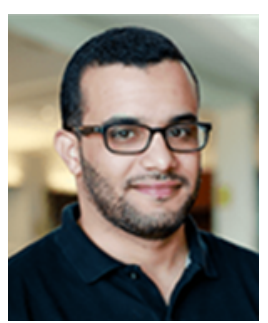

Abdeldjalil Reguig received the M.S. degree and the $\mathrm{Ph} . \mathrm{D}$. degree in 2012 and 2017 respectively, from the faculty of electrical engineering, University of Sidi Bel-Abbes, Algeria. Since 2017, he is postdoctoral fellow at the King Abdullah University of Science and Technology, in Saudi Arabia. His research mainly focus on plasma discharges at at mospheric pressure and applied electrostatics.

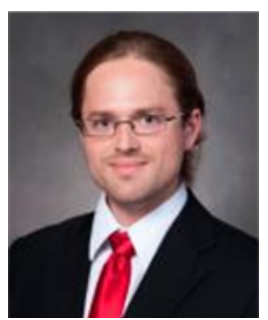

Jason Damazo graduated with a Ph.D. in Aeronautical Engineering from the California Institute of Technology where he focused on the interaction of gaseous detonations with structures. Since graduating, he has worked for The Boeing Company testing, modeling, and analyzing ignition, explosion, and other potentially high strain-rate events as they apply to aerospace product development and certification. 


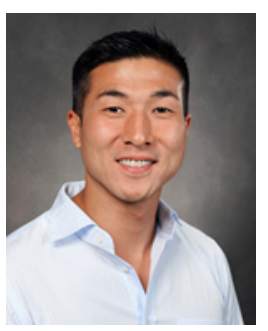

Eddie Kwon received the B.S. degree in physics from Oregon State University, USA, in 2004. From 2005 to 2019, he was researcher at the Ignition Dynamics Laboratory of The Boeing Company. Since 2020, he is the CEO of Narion Corporation. His research mainly focusses on determining the ignition threshold for novel ignition mechanisms to reduce accidental explosions in the aerospace industry.

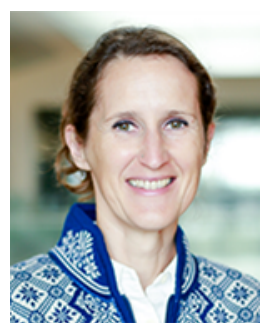

Deanna Lacoste graduated with a Ph.D. in mechanical engineering from the University of Poitiers, France, in 2002. Since 2016, she is an assistant professor of mechanical engineering at the Clean Combustion Research Center, of the King Abdullah University of Science and Technology, in Saudi Arabia. Her research mainly focus on plasma-assisted combustion, non-equilibrium plasma discharges at atmospheric pressure, control of flame dynamics and detonation. 